COMMENT. Meta-analysis (Thayyil S et al. 2010, 2008) has provided important evidence on the use of MR biomarkers for identification of neonates with moderately severe HIE who might benefit from neuroprotection or early developmental intervention. MR in isolation is not sufficiently accurate to direct treatment-limitation decisions, but used in combination with Sarnat clinical staging (1976) and electroencephalography, MRI may be more reliable. In the current report, Wilkinson provides a list of 77 references related to this topic.

\title{
NEONATAL EPISODIC LARYNGOSPASM TREATED WITH SODIUM CHANNEL BLOCKERS
}

Three male neonates with episodic laryngospasms responsive to sodium channel blockers are reported by researchers at centres in Lyon, Paris and Rennes, France. Patient 1 was admitted to an ICU at 16 days for acute life-threatening laryngospasm associated with generalized stiffness, cyanosis, and bradycardia, without loss of consciousness or startle reaction, and lasting a few minutes. Exam was normal between attacks, but repeated episodes of apnea with larngospasm, unresponsive to clonazepam or levodopa, required tracheostomy at 3 months. EMG revealed intense generalized myotonia, and at 6 months the infant had developed marked muscular hypertonia. Sequencing the SCN4A gene showed a novel heterozygous mutation. Laryngospasm was controlled with oral carbamazepine $(40 \mathrm{mg} / \mathrm{kg} / \mathrm{day})$, tracheostomy was removed at 14 months, and psychomotor development and growth were normal by 18 months of age. Patient 2 was admitted with daily episodic apneas at 3 days and intermittent stridor by 2 months. An athletic appearance developed by 7 months, and EMG showed continuous myotonia, exacerbated by cold food and winter. SCN4A analysis showed the same mutation as patient 1. Laryngospasm was controlled with mexiletine, and he could walk at 16 months. Patient 3 had immediate respiratory distress at birth, episodic stridor with apnea, and hypertonia. Spasms occurred with laryngeal endoscopy, and the patient died at 10 days. SCN4A gene sequencing showed a heterozygous de novo mutation. Gene analysis in the parents of all 3 patients was negative for SCN4A mutation. (Lion-Francois L, Mignot C, Vicart S, et al. Sevbere neonatal episodic laryngospasm due to de novo SCN4A mutations. A new treatable disorder. Neurology Aug 19, 2010;75:641-645). (Respond: Dr Laurence Lion-Francois, Hopital Femme Mere Enfant, Bron, France. E-mail: laurence.francois@chu-lyon.fr).

COMMENT. Severe neonatal episodic laryngospasm is reported as a new phenotype, associated with de novo mutations in the muscular channel gene SCN4A and myotonia responsive to sodium channel blockers. Nondystrophic myotonias related to mutations in the SCN4A gene include myotonia congenita (Thomsen disease), paramyotonia, and sodium channel myotonias (SCM). 